\title{
MAGNETIC FIELDS IN POLAR PROMINENCES
}

\author{
G. Y.SMOLKOV
}

Siberian Institute of Terrestrial Magnetism, Ionosphere and Radio Propagation, Irkutsk, U.S.S.R.

\begin{abstract}
The results of longitudinal magnetic field measurements in polar prominences (PP), obtained by Rust during 1964-1965 and by the author during 1966, 1969 and 1970, are considered. The magnetic field configuration in PP is similar to that of an arc. In opposite branches of such an arc the magnetic field has different polarities that do not coincide with the magnetic field polarity in current cycle BMR's. The magnetic field value increases as the angle between the line of sight and filament long axis grows. PP's reflect the behaviour of the border line between the magnetic fields of preceding and current cycles.
\end{abstract}

Polar prominences (PP), less than other prominences, are subjected to the influence of active regions. Therefore they are the most stable prominences (filaments). Study of the basic properties of PP inevitably leads to the conclusion that PP are located at borders between following parts of the current sunspot cycle BMR's and the same parts of the preceding sunspot cycle BMR's. In connection with this fact magnetic fields in PP's must reflect the general regularities of solar magnetic fields.

Only a relationship between their polarities may serve as a criterion of the correlation of a PP magnetic field with the underlying photospheric magnetic fields. The magnetic field value is not significant in this case. Its determination sufficiently depends on the prominence orientation and the magnetograph sensitivity. It does not change monotonically with height and along a prominence. To use this characteristic one needs measurements of the magnetic field intensity vector.

As result of the factors mentioned above, the polarity of the field in a PP must be inverse to that which is observed in BMR's of the current sunspot cycle.

During 1964-1965 Rust measured the magnetic field longitudinal component at 35 points of six PP's with a $20^{\prime \prime}$ aperture $(1966,1967)$. These measurements made it possible to find only the tendency of the PP magnetic fields to have a polarity inverse to the polarity of the magnetic field of the current cycle BMR's. In one PP the magnetic field was found equal to $0 \pm 2 \mathrm{G}$. In two others the magnetic field polarity coincided with the polarity of the current cycle BMR magnetic field. In each of them the magnetic field was measured only at one point. But the magnetic field measurement in one of them (also a single measurement) two days earlier showed the opposite polarity of the magnetic field.

The magnetic field measurements in separate points of a prominence do not permit us to obtain the magnetic field distribution picture. Therefore the presence of both polarities in one of the prominences led Rust to the conclusion of an extremely complicated picture of the spatial distribution of magnetic fields in prominences.

Independent measurements of magnetic fields in quiet prominences (QP) with the help of the SibIZMIR magnetograph (Kuznetzov et al., 1966) were started by the author in January, 1966 at Sayan Mountain Observatory (Smolkov, 1966). These observations made it possible to obtain a two-dimensional continuous distribution 
of the longitudinal component of the magnetic field vector in a whole prominence (Smolkov, 1967a, b). The results of measurements in 1966 showed that the magnetic field configuration in QP's and PP's may be that of an arc. The magnetic field polarity in opposite branches of such an arc may or may not coincide with the magnetic field polarity in a current cycle BMR. An example of such magnetic field distribution is given in Figure 1.

The unipolar distribution of the QP magnetic fields corresponds to cases when the magnetic field of one of the polarities is behind the limb or at the Sun's disk or when the resulting field of one polarity is measured.

Magnetic fields in PP's were measured by the author additionally in 1969 and 1970. The total number of PP's measured is equal to 12 , the number of days on which magnetic field measurements were made is equal to 25 (in 1966 correspondingly 7 and 13). In the majority of cases PP's were scanned completely one or several times a day. In some PP's the magnetic field distribution was recorded during several days one after the other or was repeated at the other limb or in another solar rotation.

The magnetic field measurements in PP's made by Rust and the author cover nearly all years from the minimum to the maximum of the current cycle. To consider the magnetic field polarity question we included in addition high-latitude old quiet

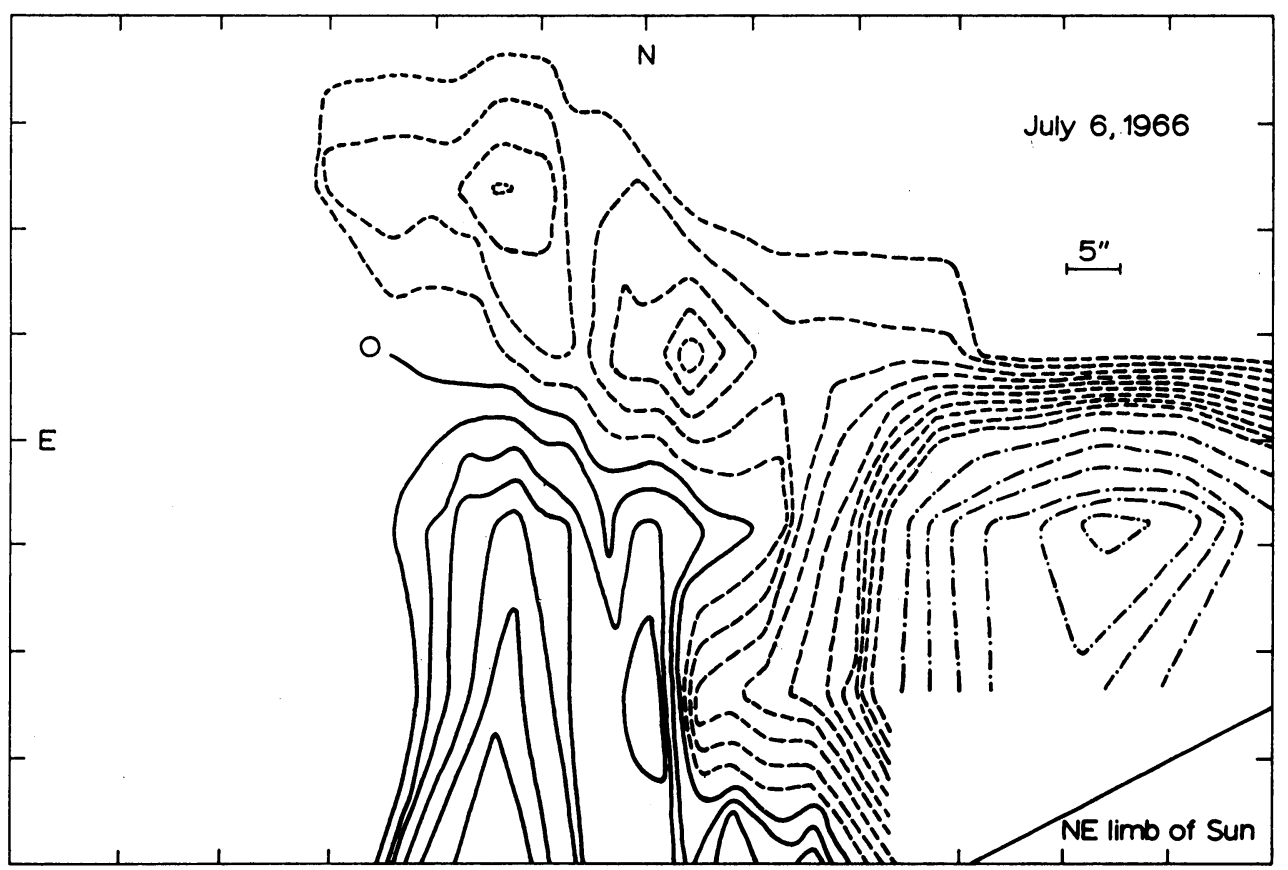

Fig. 1. Magnetogram, obtained for the Polar Prominence of July 6, 1966. Prominence coordinates $61^{\circ} \mathrm{N} 83^{\circ} \mathrm{E}$. Dashed contours enclose negative fields, solid contours enclose positive fields. Isolines on the magnetogram are at 3,6,9... G levels. Dash-dot isolines corresponds to interval of $9 \mathrm{G}$. Thick line means zero field. Standard error is close to $1 \mathrm{G}$. Scanning aperture is $0^{\prime \prime} .6 \times 5^{\prime \prime}$. The prominence orientation at the moment of magnetic field measurements conforms to the western part of the filament on the diagram of Figure 2. 
prominences (OQP). The total number of prominences considered is equal to 27 and the number of days with magnetic field measurements is equal to 47 . The total number of cases of lack of magnetic field polarity coincidence with the BMR magnetic field polarity of the current cycle before discussion was equal to $77 \%$ of the number of observation days and $76 \%$ of the number of all prominences.

Each of these prominences was considered using the Meudon synoptic charts of the solar chromosphere and the Atlas of solar magnetic fields (Howard et al., 1967). Unfortunately the Atlas covered generally only latitudes between $-40^{\circ}$ and $+40^{\circ}$ and only the years 1959-1966. A minimum value of the magnetic field on these maps is equal to $6 \mathrm{G}$. It is not possible to define the photospheric magnetic field distribution for all the prominences considered. However the overwhelming majority of the prominences indicate a scheme of the magnetic field polarities in a OQP which is discussed below. The measurements of the magnetic fields in prominences in 1969 and 1970 conform to this scheme generally. But because just now the author has no synoptic maps of the chromosphere and of the photospheric magnetic fields these measurements require additional study.

The polarity of the recorded magnetic field in high-latitude prominences depends on the long-axis orientation of a prominence (filament) in respect to the line of sight. Filaments which have a relatively large life time stretch along the heliographic latitude. At the later stage of development of filaments their eastern parts become inclined to the equator like rounding the large-scale magnetic field features.

The scheme of a developed OQP orientation in the northern hemisphere is presented in Figure 2. Measuring the magnetic fields in the western part of the filament we obtain the magnetic field polarity coinciding with the magnetic field polarity of the current cycle BMR. In the highest latitude point of filament $A$ the longitudinal magnetic field may be small and less than the magnetograph sensitivity level or may be absent altogether. In the eastern part the orientation of the photospheric magnetic

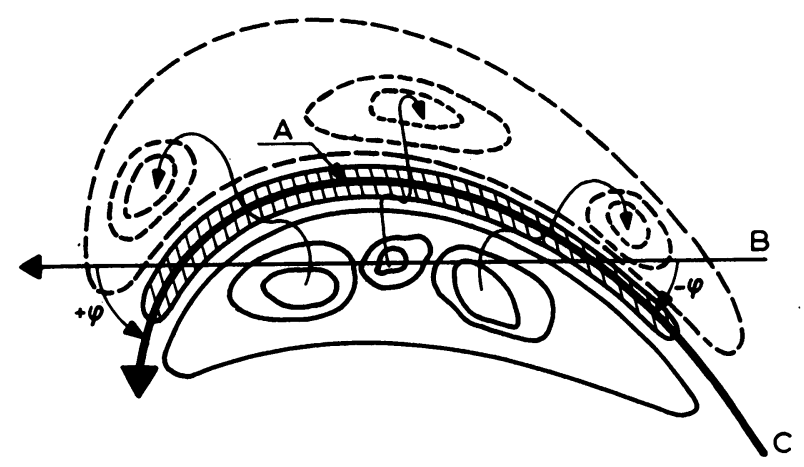

Fig. 2. Diagram of magnetic field polarity in old quiescent filaments for the northern hemisphere. Filaments divide the following patches of BMR preceding and current sunspots cycles. Dashed contours enclose negative fields, solid contours enclose positive fields. $\varphi$ is angle between the line of sight and the filament long axis. A negative angle means the coincidence of the polarity of the magnetic field of old quiescent filaments and of a current cycle BMR, a positive angle is of the opposite sense. The point $A$ dividing the filament in the eastern and western parts is closest to the pole. The letter $B$ means the line of sight. The letter $C$ means the filament long axis. 
field force lines becomes inverse. Therefore the author in the case of such a filament orientation recorded a magnetic field polarity which does not coincide with the magnetic field polarity in the current cycle BMR's, as did Rust.

For PP's having a primary orientation along a heliographic latitude, such conditions of the magnetic field measurements arise as a result of the inclination of the Sun's rotation axis.

The magnetic field in two prominences of 1964-1965 (December 17, 1964 and September 27, 1965) and in one prominence of 1966 (April 2, 1966) deviated from this rule. In all these cases owing to the development of new BMR's, local temporary inhomogeneities of the magnetic field were formed which changed its polarity into an inverse one temporarily. In both cases of the first set, the photospheric magnetic field distribution had been restored by the next solar rotation. The filaments were changed after this. The prominence of 1966 disappeared soon.

All the rest of the prominences of 1964-1966 had a magnetic field polarity strictly corresponding to Figure 2.

The inversion of the magnetic field polarity when passing the point $A$ (Figure 2) and the null value of the magnetic field in such parts of the filament testify to the predominance of the magnetic field directed for the most part perpendicularly to the long axis filament. During the measurements in the same prominence, the magnetic field value increases as the angle between the sight line and its long axis grows. These facts show that OQP's have no magnetic fields of their own. Using the magnetograph measurements one records the magnetic field caused by photospheric sources which penetrate into the prominence. This is in agreement with the theory of filaments by Kippenhahn and Schlüter (1957).

The results obtained confirm the close connection of OQP's with the photospheric magnetic fields. One may consider that PP's reflect the behaviour of the line of division of the magnetic fields of preceding and current cycles. This was shown theoretically by Leighton (1969). The coincidence of the differential rotation velocity of the photospheric magnetic fields with the filament rotation velocity found by Wilcox and Howard (1970) may serve as an additional reason.

\section{References}

Howard, R., Bumba, V., and Smith, S. F.: 1967, Carnegie Institution of Washington Publication, No. 626.

Kippenhahn, R. and Schlüter, A.: 1957, Z. Astrophys. 42, 36.

Kuznetzov, D. A., Kuklin, G. V., and Stepanov, V. E.: 1966, 'Results of Observations and Researches in Period IQSY. Siberia and Far East. I', Solar Physics and Cosmic Rays, p. 80.

Leighton, R. B.: 1969, Astrophys. J. 156, 1.

Rust, D. M.: 1966, Ph.D. Thesis, Univ. of Colorado.

Rust, D. M.: 1967, Astrophys. J. 156, 313.

Smolkov, G. Y.: 1966, Researches on Geomagnetism and Aeronomy p. 189.

Smolkov, G. Y.: 1967a, 'Results of Observations and Researches in Period IQSY. Siberia and Far East, II', Solar Physics and Cosmic Rays, in press.

Smolkov, G. Y.: 1967b, 'Results of Observations and Researches in Period IQSY. Siberia and Far East, II', Solar Physics and Cosmic Rays, in press.

Wilcox, J. M. and Howard, R.: 1970, Solar Phys. 13, 251. 